\title{
Fuzzy Sets for Image Processing Applications: Deleting Objects
}

\author{
${ }^{1}$ Noor Riyadh Kareem and Hind Rustum Mohammed \\ ${ }^{1}$ Department of Mathematics, \\ ${ }^{2}$ Department of Computer Science, Faculty of Computer Science and Maths, \\ University of Kufa, Kufa, Iraq
}

\begin{abstract}
In this research, a new method for deleting objects for colour images based on fuzzy sets are presented. The idea of filtering is that the fuzzy set filter to remove objects. The results show that the proposed filter is more efficient than other methods. MATLAB would be the paper environment (data set are 30 colour images with any format). Performance criteria used to demonstrate the accuracy of the method used and its best statistical criteria such as (mean and peak signal to noise ratio).
\end{abstract}

Key words: Fuzzy set, fuzzy point, quasi-coincident, fuzzy net, image processing, image objects, image filtering, statistical parameters

\section{INTRODUCTION}

In 1965 , first publications in fuzzy set theory by Zadeh (Nouh, 2005) which encouraged the researchers to generalize the classical notion of a set. After many years by Pao-Ming and Ying-Ming (1980) were devoted the concept of q-coincident and fuzzy net. The only sets which can be handled on computers are discrete or digital sets which means sets that contain at most a denumerable number of elements.

The fuzzy rule base algorithm presented in this research in the environment of MATLAB which is capable of deleting small and large size objects for colour images.

Fuzzy set: The concept of fuzzy set and several properties of it introduced in this study which are needed in our research.

Definition 2.1: Zadeh (1965): A fuzzy set $\mathrm{k}$ in $\mathrm{x}$ is a function from a non-empty set $\mathrm{x}$ into the unit closed Interval $\mathrm{I}$ where $\mathrm{I}=(0,1)$. (i.e., $\mathrm{k}: \mathrm{x} \rightarrow \mathrm{I}$ be a function). For all $\mathrm{a} \in \mathrm{X}$, the degree of membership of element $\mathrm{a}$ in a fuzzy set $\mathrm{k}$ it is the interpretation of $\mathrm{k}(\mathrm{a})$.

The set of pairs: $\mathrm{k}=\{\{\mathrm{a}, \mathrm{k}(\mathrm{a})\}): \mathrm{a} \in \mathrm{x}\}$ is one of the ways to express of a fuzzy subset $k$ of $x$. The class of all fuzzy subsets of a set $\mathrm{x}$ is denoted by $\mathrm{I}^{\mathrm{x}}$.

Definition 2.2; Nouh (2005) and Pao-Ming and Ying-Ming (1980): If $\mathrm{k}$ and $\mathrm{H}$ are fuzzy subsets of a set $\mathrm{x}$. Then, we put:

- $k=\mathrm{H}$ if and only if $\mathrm{k}(\mathrm{a})=\mathrm{H}$ (a) for every $\mathrm{a} \in \mathrm{x}$

- $\mathrm{k} \leq \mathrm{H}$ if and only if $\mathrm{k}(\mathrm{a}) \leq \mathrm{H}$ (a), for every $\mathrm{a} \in \mathrm{x}$
- $\quad z=k \vee H$ if and only if $z(a)=\max \{k(a), H(a)\}$ for every $\mathrm{a} \in \mathrm{x}$

- $\quad \mathrm{D}=\mathrm{k} \wedge \mathrm{H}$ if and only if $\mathrm{D}(\mathrm{a})=\min \{\mathrm{k}(\mathrm{a}), \mathrm{H}(\mathrm{a})\}$ for every a $\in \mathrm{X}$

- $\quad \mathrm{F}=\mathrm{k}^{\mathrm{c}}$ if and only if $\mathrm{F}(\mathrm{a})=1-\mathrm{k}(\mathrm{a})$, for every $\mathrm{a} \in \mathrm{x}$

Definition 2.3: Pao-Ming and Ying-Ming (1980): A fuzzy (singleton) point in $\mathrm{x}$ is a fuzzy subset of $\mathrm{x}$ defined by:

$$
z_{r}(y)=\left\{\begin{array}{l}
r, \text { if } y=z, \\
0, \text { otherwise }
\end{array}\right.
$$

For each $z \in \mathrm{x}$, the support of $\mathrm{z}_{\mathrm{r}}$ is the single point $\mathrm{z}$ and $\mathrm{r} \in\{0,1\}$ is said to be its value. $\mathrm{FP}(\mathrm{x})$ denote to the family of all fuzzy points in $\mathrm{x}$. A fuzzy point $\mathrm{z}_{\mathrm{r}}$ is said to be belongs to a fuzzy set $k$ or $z_{r}$ contained in $k$, i.e., $z_{r} \in k$ if and only if $r \leq k(z)$. Two fuzzy points $z_{s}$ and $y_{s}$ in $x$ are named to be distinct if and only if their supports are distinct.

Definition 2.4: Pao-Ming and Ying-Ming (1980): A fuzzy subset $\mathrm{k}$ of $\mathrm{x}$ is called quasi-coincident (in short qcoincident) with a fuzzy subset $\mathrm{H}$ of $\mathrm{x}$, denoted by $\mathrm{kqH}$ if and only if $\mathrm{k}(\mathrm{a})+\mathrm{H}(\mathrm{a})>1$, for some $\mathrm{a} \in \mathrm{x}$. Otherwise, then, $\mathrm{k}$ is said to be not q-coincident with $\mathrm{H}$ and it is denoted by $\mathrm{kqH}$, i.e., $\mathrm{k}(\mathrm{a})+\mathrm{H}(\mathrm{a}) \leq 1$ for each $\mathrm{a} \in \mathrm{x}$.

Definition 2.5: Pao-Ming and Ying-Ming (1980): A fuzzy point $z_{r}$ in $x$ is named q-coincident with a fuzzy subset $\mathrm{k}$ of $\mathrm{x}$, denoted by $\mathrm{z}_{\mathrm{q}} \mathrm{k}$ if and only if $\mathrm{r}+\mathrm{k}(\mathrm{z})>1$.

Remark 2.6: Pao-Ming and Ying-Ming (1980): If $z_{r} \in F P(x)$ and $\mathrm{k}$ is a fuzzy set in a set $\mathrm{x}$. Then:

Corresponding Author: Noor Riyadh Kareem, Department of Mathematics, Faculty of Computer Science and Maths, Faculty of Computer Science and Maths, University of Kufa, Kufa, Iraq 


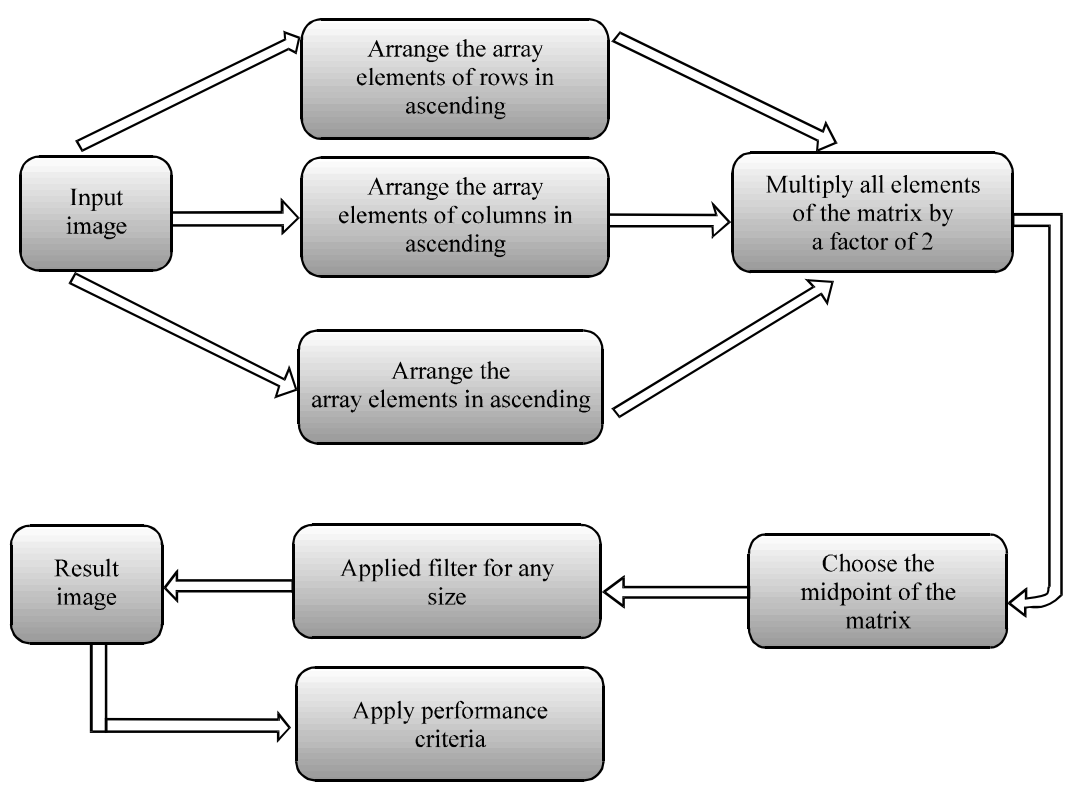

Fig. 1: Pixel level image fusion using fuzzy set

- $\quad \mathrm{z}_{\mathrm{r}} \mathrm{qk}$ if and only if $\mathrm{r}>\mathrm{k}^{\mathrm{c}}(\mathrm{z})$

- $\quad z_{\mathrm{r}} \tilde{\mathrm{q} k \mathrm{k}}$ if and only if $\mathrm{r} \leq \mathrm{k}^{\mathrm{c}}(\mathrm{z})$

Lemma 2.7: Pao-Ming and Ying-Ming (1980): For any fuzzy point $\mathrm{z}_{\mathrm{i}}$ in $\mathrm{x}$ and for $\mathrm{k}$ any fuzzy set in $\mathrm{x}$. Then, the following statements are equivalent:

- $\mathrm{z}_{\mathrm{r}} \in \mathrm{k}$

- $\mathrm{z}_{\mathrm{r}} \tilde{\mathrm{k}} \mathrm{k}^{\mathrm{c}}$

- $z_{1-r} q k$

Definition 2.8: Pao-Ming and Ying-Ming (1980): A directed $\operatorname{set}(\mathrm{D}, \geq)$ is a set $\mathrm{D}$ with a relation $\geq$ satisfying:

- $\mathrm{n} \geq \mathrm{n}$ for each $\mathrm{n} \in \mathrm{D}$

- If $n_{1} \geq n_{2}$ and $n_{2} \geq n_{2}$, then $n_{1} \geq n_{3}$

- If $n_{1}, n_{2} \in D$, then, there is some $n_{3} \in D$ with $n_{3} \geq n_{1}$ and $\mathrm{n}_{3} \geq \mathrm{n}_{2}$

Definition 2.9: Nouh (2005) and Pao-Ming and Ying-Ming (1980): A fuzzy net $\mathrm{f}$ in a set $\mathrm{x}$ is denoted by $\{s(n): n \in D\}$ and defined as the mapping from directed set $\mathrm{D}$ to fuzzy point in $\mathrm{x}$, i.e., $\mathrm{F}: \mathrm{D} \rightarrow \mathrm{FP}$ ( $\mathrm{x}$ ). If $\mathrm{s}$ (n) $=\mathrm{s}(\mathrm{n})=\mathrm{z}_{\mathrm{m}}^{\mathrm{n}}$ for each $\mathrm{n} \in \mathrm{D}$ where $\mathrm{z} \in \mathrm{X}, \mathrm{n} \in \mathrm{D}$ and $\mathrm{r}_{\mathrm{n}} \in(0,1)$, then, the Fuzzy net $F$ is denoted as $\left\{z_{\mathrm{m}}^{\mathrm{n}}: \mathrm{n} \in \mathrm{D}\right\}$ or simply $\left\{\mathrm{z}_{\mathrm{m}}^{\mathrm{n}}\right\}$.

Definition 2.10: Pao-Ming and Ying-Ming (1980): If $\mathrm{F}=\left\{\mathrm{Z}_{\mathrm{m}}^{\mathrm{n}}: \mathrm{n} \in \mathrm{D}\right\}$ is a fuzzy net in a set $\mathrm{x}$ and $\mathrm{k}$ is a fuzzy subset of $\mathrm{x}$. Then, $\mathrm{F}$ is said to be:
- Q-eventually with $\mathrm{k}$ if and only if $\exists \mathrm{m} \in \mathrm{D}$ such that $z_{\mathrm{m}}^{\mathrm{n}} \mathrm{qk}, \forall \mathrm{n} \geq \mathrm{m}$

- Q-frequently with $\mathrm{k}$ if and only if $\forall \mathrm{n} \in \mathrm{D}, \exists \mathrm{m} \in \mathrm{D}$ with $\mathrm{m} \geq \mathrm{n}$, such that $\mathrm{z}_{\mathrm{m}}^{\mathrm{m}} \mathrm{qk}$

\section{MATERIALS AND METHODS}

The steps algorithm for using fuzzy set is given as follows Algorithm 1:

\section{Algorithm 1; Fuzzy set algorithm:}

Step 1: Read colour images and converting them within the closed period $(0,1)$

Step 2: (a) (Mask 1) arrange the array elements of rows in ascending order according to their blur values

(b) (Mask 2) Arrange the array elements of columns in ascending order according to their blur values

(c) (Mask 3) Arrange the array elements of rows and columns at the same time in ascending order according to their blur values

Step 3: Multiply all elements of the matrix by a factor of 2

Step 4: Choose the midpoint of the matrix for each applied filter for any size Step 5: Find final image which more deleting objects

Step 6: Print all images with any filter fuzzy set matrix and final image

Step 7: Apply performance criteria to determine the best steps such as (mean and peak signal to noise ratio)

Figure 1 showed diagram for pixel level image fusion using fuzzy set system

\section{RESULTS AND DISCUSSION}

After reading the image within the blur values and converting them within the closed period $(0,1)$ and arranging values of matrix in ascending order which is a basic condition in our research. We used three types of arrangement of matrix elements as follows: 

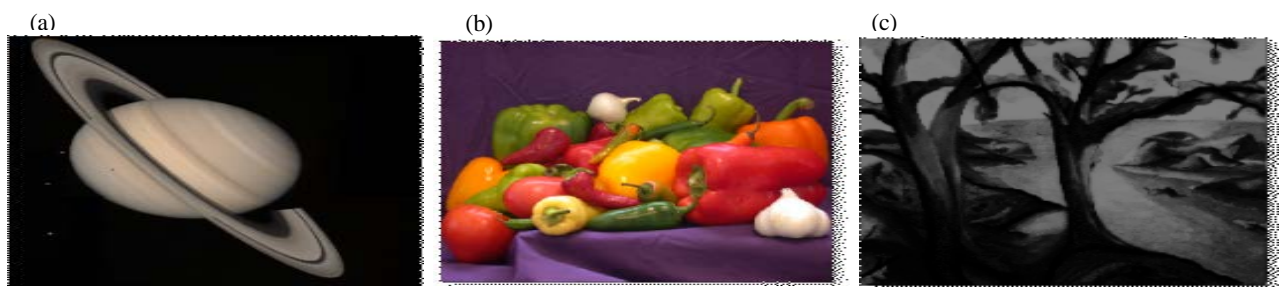

Fig. 2: Sample of colour images: a) Image 1; b) Image 2 and c) Image 3
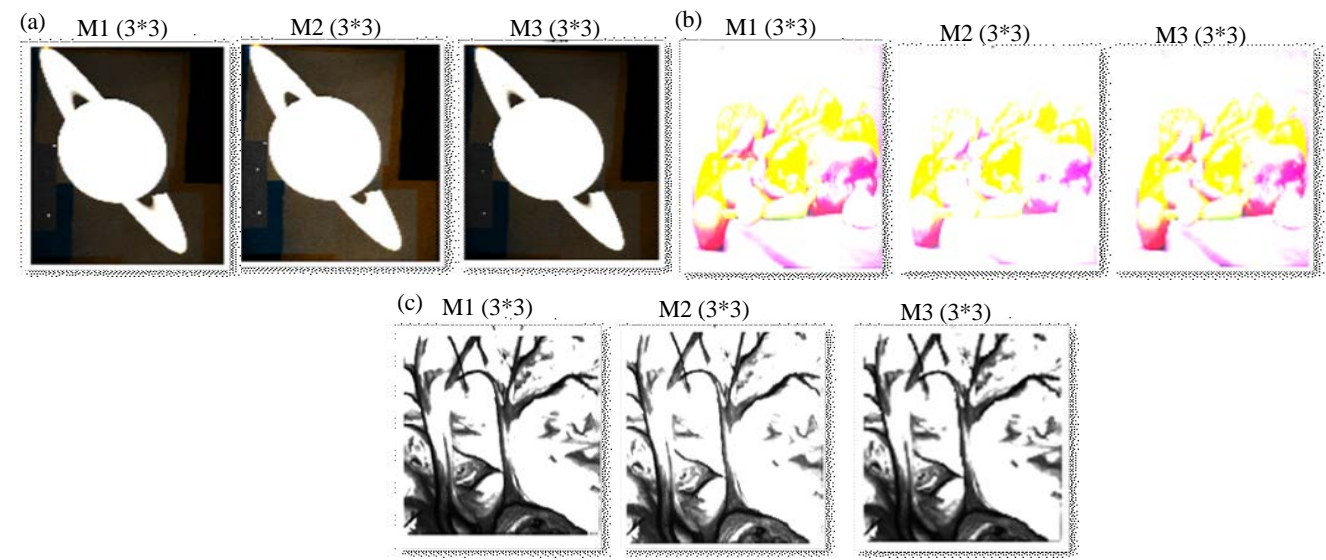

\section{M2 (3*3)}
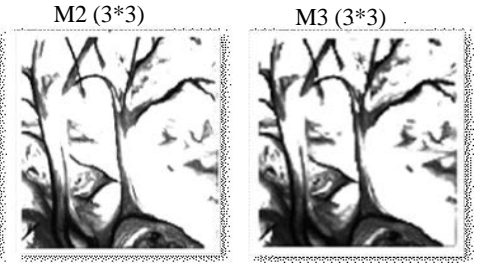

Fig. 3: a-c) Effect for deleting objects based on mask size ( $\left.3^{*} 3\right)$ for colour images
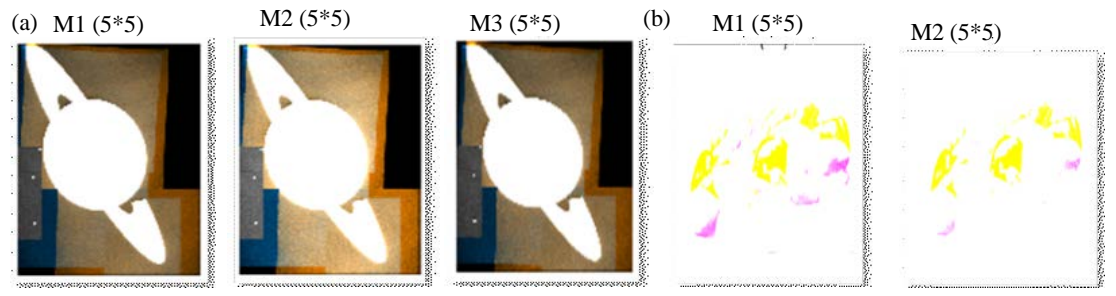

M3 (5*5)
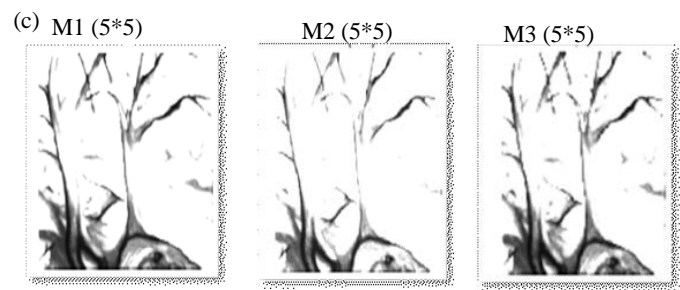

Fig. 4: a-c) Effect for deleting objects based on mask size $\left(5^{*} 5\right)$ for colour images

- Arrange values of matrix in ascending order according to rows only

- Arrange values of matrix ascending order by columns only

- Arrange values of matrix by rows and columns at the same time

Where we considered that each of the above arrangements is a mask, hit all elements of the matrix by factor 2. We choose the middle point of the matrix to apply the filters of any size (Eckhardt and Latecki, 1994; Tang, 2004).
Figure 2 shown sample of colour images when the system was executed on a database of 30 colour images with any size and any format (Fig. 3-6).

The application of the above mentioned law where we observe in the resultant values whose values are higher than 1 of a given point and within the chosen order, evidence that the law is validated as stated in definition (2.10), the first case Q-eventually. In the case of the application of the law, the output was higher than 1 for some points and within the chosen order, a proof that the law is verified as stated in the definition (2.10). The second case Q-frequently (Table 1). 


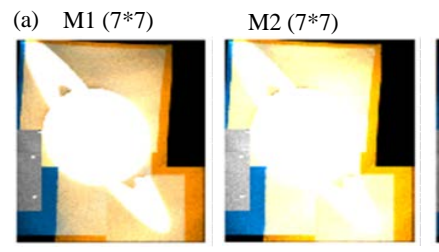

(c) $\mathrm{M} 1\left(7^{* 7)}\right.$

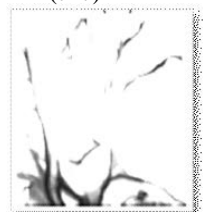

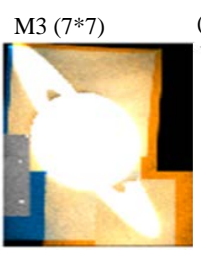

M2 (7*7) (b) $\mathrm{M} 1(7 * 7)$

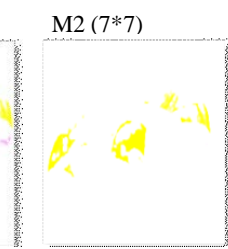

M3 (7*7)

M3 (7*7)
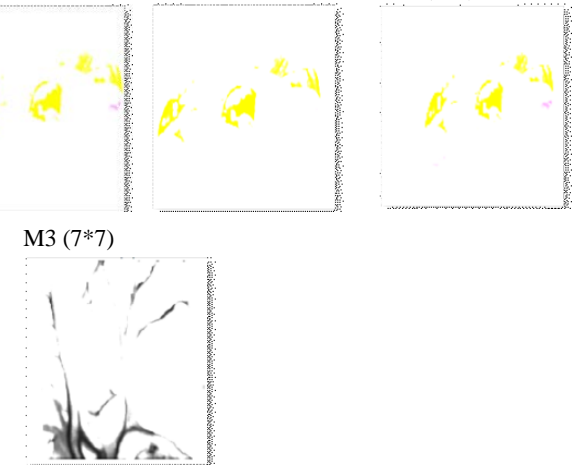

Fig. 5: a-c) Effect for deleting objects based on mask size $\left(7^{*} 7\right)$ for colour images

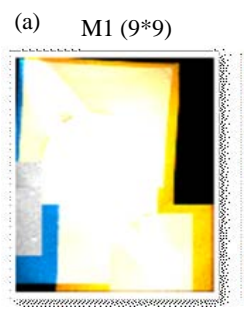

(b)

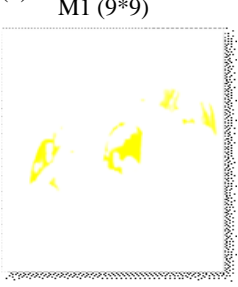

(c) $\mathrm{M} 1(9 * 9)$

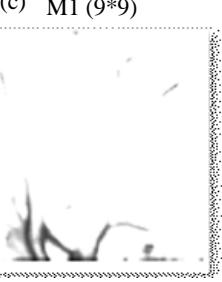

M1 (9*9)

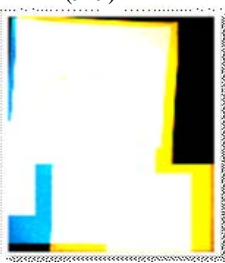

M1 (9*9)

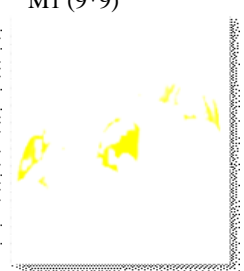

M1 (9*9)

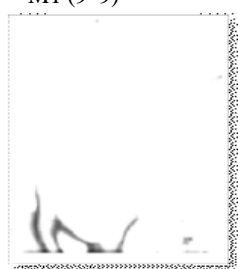

M1 (9*9)

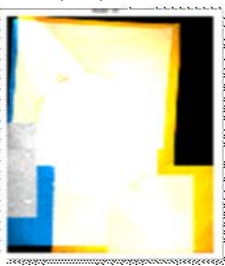

M1 (9*9)

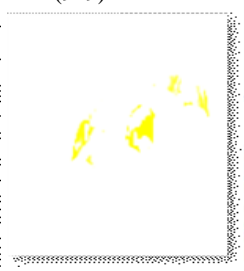

M1 (9*9)

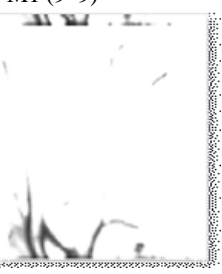

(d)

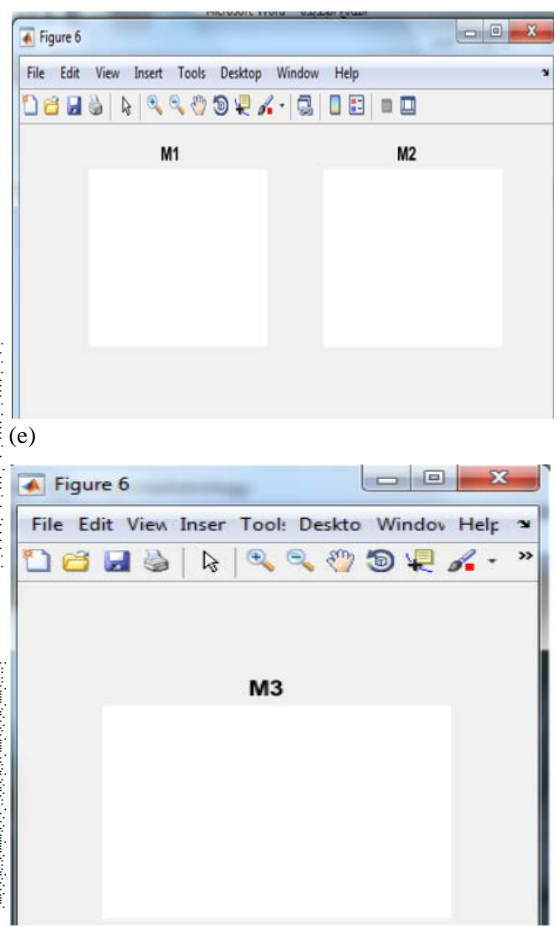

Fig. 6: a-c) Effect for deleting objects based on mask size (9*9) for colour images

Table: 1 Statistical features (mean and peak signal to noise ratio) for output images

\begin{tabular}{|c|c|c|c|c|c|c|}
\hline \multirow[b]{2}{*}{ Statistical tools/Masks } & \multicolumn{3}{|l|}{ Mean } & \multicolumn{3}{|c|}{ Peak signal-to-noise ratios } \\
\hline & Image 1 & Image 2 & Image 3 & Image 1 & Image 2 & Image 3 \\
\hline $\operatorname{Mask} 1(3 * 3)$ & 95.6764 & 237.4879 & 177.1953 & 10.1349 & 3.6415 & 5.0786 \\
\hline $\operatorname{Mask} 2(3 * 3)$ & 105.7345 & 243.4286 & 194.5291 & 9.4738 & 3.3493 & 4.2217 \\
\hline $\operatorname{Mask} 3(3 * 3)$ & 95.4036 & 237.3156 & 175.6096 & 10.1535 & 3.6513 & 5.1463 \\
\hline Mask1 $(5 * 5)$ & 126.0526 & 247.9592 & 220.5644 & 7.8912 & 3.1437 & 3.0788 \\
\hline $\operatorname{Mask} 2(5 * 5)$ & 150.6799 & 249.1151 & 233.2927 & 5.8797 & 3.0874 & 2.5464 \\
\hline Mask3(5*5) & 125.8274 & 247.5479 & 219.9954 & 7.9093 & 3.1577 & 3.1026 \\
\hline $\operatorname{Mask} 1(7 * 7)$ & 168.4957 & 250.1953 & 241.4365 & 4.5703 & 3.0451 & 2.2301 \\
\hline $\operatorname{Mask} 2(7 * 7)$ & 191.0463 & 250.5381 & 247.1375 & 3.2253 & 3.0300 & 2.0097 \\
\hline $\operatorname{Mask} 3(7 * 7)$ & 168.3119 & 250.1711 & 241.4158 & 4.5826 & 3.0471 & 2.2303 \\
\hline $\operatorname{Mask} 1(9 * 9)$ & 195.7729 & 251.7534 & 249.5529 & 2.9693 & 2.9942 & 1.9199 \\
\hline $\operatorname{Mask} 2(9 * 9)$ & 204.2788 & 251.7952 & 251.4369 & 2.5199 & 2.9837 & 1.8442 \\
\hline Mask3(9*9) & 195.7484 & 251.3783 & 249.1419 & 2.9707 & 3.0010 & 1.9342 \\
\hline
\end{tabular}


Both the mean and the peak single to noise ratio used on output images, the result were as shown in the following Table 1.

\section{CONCLUSION}

In this research we used effect method for deleting objects for colour images based on fuzzy sets and we represented values of the fuzzy sets and extracted the figures and histogram of the characteristics for images. Note that fuzzy sets filters are efficiency in deleting and hiding objects in colour images is very high. More deleting objects when increased size of fuzzy sets filters and when used factor equal 2. Our program can use in image security with any types of colour images and any format.

When we applying the mean on images, we found that the values of the second mask were the highest while the values of the third mask were the lowest and the values of the first mask were between those two values.

As well as when applying the peak single to noise ratio on images, we found that the values of the third mask were the highest while the values of the second mask were the lowest and the values of the first mask were between those two values.
However, when applying some other statistical parameters such as (entropy, standard division, structural similarity index) on images, we found that mask values are various.

\section{REFERENCES}

Eckhardt, U. and L. Latecki, 1994. Digital topology. Master Thesis, University of Hamburg, Council of Scientific Information, Vilayil Gardens, Trivandrum, India.

Nouh, A.A., 2005. On convergence theory in fuzzy topological spaces and its applications. Czech. Math. J., 55: 295-316.

Pao-Ming, P. and L. Ying-Ming, 1980. Fuzzy topological I. neighborhood structure of a fuzzy point and Moore-Smith convergence. J. Math. Anal. Appl., 76: 571-599.

Tang, X., 2004. Spatial object modeling in fuzzy topological spaces with applications to land cover change in China. Ph.D Thesis, University of Twente, Enschede, Netherlands.

Zadeh, L.A., 1965. Fuzzy sets. Inform. Control, 8: 338-353. 